\title{
Pattern Discovery and Listing in Graphs
}

\author{
Roberto Grossi \\ Dipartimento di Informatica \\ Università di Pisa \\ grossi@di.unipi.it
}

Graphs are gaining increasing popularity in many application domains as they have the potential of modeling binary relations among entities. Along with textual and multimedia data, they are the main sources for producing large data sets. It is natural to ask how it is easy to extend the notion of patterns typically found in string matching and sequence analysis, to graphs and real-life networks. Unfortunately, even the basic problem of finding a simple path in a graph is NP-hard since this can establish if the graph is Hamiltonian. Also, the number of patterns can be exponentially large in the size of the graph, thus listing them is a challenge. We will discuss some output-sensitive and parameterized algorithms for listings patterns that are paths, cycles and trees, and provide a notion of "certificate" to attain this goal. This is joint work with Rui Ferreira.

The original version of this chapter was revised: The copyright line was incorrect. This has been corrected. The Erratum to this chapter is available at DOI: 10.1007/978-3-319-02432-5_33

O. Kurland, M. Lewenstein, and E. Porat (Eds.): SPIRE 2013, LNCS 8214, p. 2, 2013.

(C) Springer-Verlag Berlin Heidelberg 2013 